\title{
Hydrocarbon dust absorption in Seyfert galaxies and ULIRGs
}

\author{
R. E. Mason ${ }^{1} \dagger$, G. S. Wright ${ }^{2}$, Y. J. Pendleton ${ }^{3}$ \\ and A. J. Adamson ${ }^{4}$ \\ ${ }^{1}$ Institute for Astronomy, Royal Observatory Edinburgh, Blackford Hill, Edinburgh \\ EH9 3HJ, UK email: rmason@noao.edu \\ ${ }^{2}$ UK Astronomy Technology Centre, Royal Observatory Edinburgh, Blackford Hill, Edinburgh, \\ EH9 3HJ, UK \\ ${ }^{3}$ NASA Ames Research Center, Moffet Field, CA 90345, USA \\ ${ }^{4}$ Joint Astronomy Centre, 660 North A'ohoku Place, University Park, Hilo, HI 96720, USA
}

\begin{abstract}
We present new spectroscopic observations of the $3.4 \mu \mathrm{m}$ absorption feature in a Seyfert 2 galaxy and a ULIRG. A signature of $\mathrm{C}-\mathrm{H}$ bonds in aliphatic hydrocarbons, the $3.4 \mu \mathrm{m}$ feature indicates the presence of organic material in Galactic and extragalactic dust. The feature in these galaxies closely resembles that seen in the Galactic diffuse ISM and in newly-formed dust in a protoplanetary nebula. This similarity implies a common composition for the hydrocarbon component of interstellar dust in a range of galaxy types, and one which is resistant to processing in the interstellar and/or circumnuclear medium.
\end{abstract}

A useful tracer of the carbonaceous component of interstellar dust is the $\mathrm{C}-\mathrm{H}$ bond stretch of aliphatic hydrocarbons, seen in absorption at $3.4 \mu \mathrm{m}$ throughout the Galactic diffuse ISM. This feature has structure arising from symmetric/asymmetric stretches of $\mathrm{C}-\mathrm{H}$ bonds in $\mathrm{CH}_{2}$ and $\mathrm{CH}_{3}$ groups in hydrocarbon chains, the relative strengths of which relate to the lengths of the chains. Also, chemical groups such as $\mathrm{CO}, \mathrm{CN}$, and $\mathrm{OH}$ attached to the chains can change the force constants of the $\mathrm{C}-\mathrm{H}$ bonds, causing shifts in the positions and intensities of the subpeaks in the band. The overall shape of the $3.4 \mu \mathrm{m}$ feature reflects this and can therefore be used to probe the chemical makeup of the dust.

To extend the range of environments in which interstellar hydrocarbons are observed, we have begun to obtain spectra of the $3.4 \mu \mathrm{m}$ feature towards the nuclei of Seyfert galaxies and ULIRGs (galaxies with $L_{I R}>10^{12} L_{\odot}$; Mason et al. 2004). Fig. 1 compares the shape of the feature in NGC1068, the archetypal Seyfert 2 galaxy, with the Galactic diffuse ISM, newly-formed dust in a protoplanetary nebula, and with the ULIRG, IRAS08572. The similarity of the feature profile in each case is striking. Not only are the peak wavelengths good matches, but the relative strengths of the subfeatures are also very similar between all the lines of sight. The $3.4 \mu \mathrm{m}$ feature in a third ULIRG, IRAS19254, has also been found to have a similar profile (Risaliti et al. 2003).

Based on fits to the Galactic $3.4 \mu \mathrm{m}$ feature, a number of dust analogue materials produced by very different mechanisms have been suggested to represent the carbonaceous component of dust. For example, certain processed ice residues can produce as good a match to the interstellar feature as can residues made from carbon plasmas (Pendleton et al. 1994). However, there also exist dust candidate materials with varied $3.4 \mu \mathrm{m}$ bands which differ clearly from the astronomical spectra (Fig. 1). If the dust in other galaxies were formed by a different mechanism from Galactic dust and/or underwent different

$\dagger$ Present Address: NOAO Gemini Science Center, 950 North Cherry Ave., Tucson, AZ85719, USA 

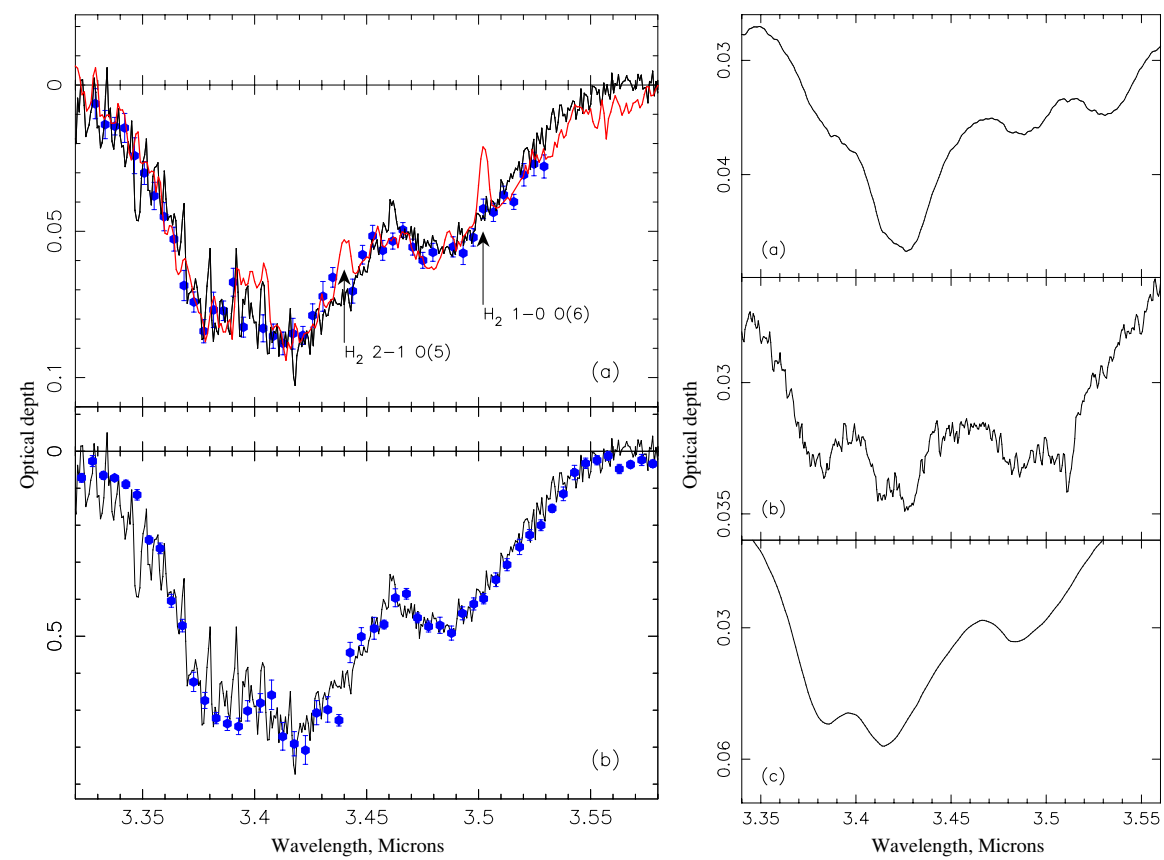

Figure 1. Left: (a) The $3.4 \mu \mathrm{m}$ feature in NGC1068 (black line) compared with that in the diffuse ISM towards the Galactic centre (Pendleton et al. 1994, points) and the protoplanetary nebula, CRL618 (Chiar et al. 1998, grey line); (b) The feature in NGC1068 compared with that in IRAS08572+3915. Right: The $3.4 \mu \mathrm{m}$ band can vary markedly in profile. (a) A quenched carbonaceous condensate (Sakata et al. 1987); (b) A UV-irradiated ice (Allamandola, Sandford \& Valero 1988);(c) An interstellar HAC analogue (Furton et al. 1999).

processing, then variations in the $3.4 \mu \mathrm{m}$ band profile could certainly arise. Instead, we observe that the bands in these galaxies are a very good match to each other and to Galactic spectra along many different sightlines. Rather than different processes which coincidentally produce materials with almost identical $3.4 \mu \mathrm{m}$ bands, the similarity of the feature suggests similar dust formation and processing mechanisms to those responsible for the feature in the Galactic diffuse ISM.

Given the variation in environmental conditions in these lines of sight (AGN/starformation activity, shielding column densities, UV/X-ray fluxes etc.), this uniformity is intriguing. It also implies that the hydrocarbon chains in dust are very robust. High-S/N spectra of a larger galaxy sample will show whether this remarkable chemical similarity is common to active, ultraluminous and quiescent galaxies alike.

\section{References}

Allamandola, L. J., Sandford, S. A., \& Valero, G. J. 1988, Icarus, 76, 225

Chiar, J. E., Pendleton, Y. J., Geballe, T. R., \& Tielens, A. G. G. M. 1998, ApJ, 507, 281

Furton, D. G., Laiho, J. W., \& Witt, A. N. 1999, ApJ, 526, 752

Mason, R. E., Wright, G. S., Pendleton, Y. J., \& Adamson, A. J. 2004, in prep

Pendleton, Y. J., Sandford, S. A., Allamandola, L. J., Tielens, A. G. G. M., \& Sellgren, K. 1994, ApJ, 437, 683

Risaliti, G., et al. 2003, ApJ, 595, L17

Sakata, A., Wada, S., Onaka, T., \& Tokunaga, A. 1987, ApJ, 320, L63 